PROCEEDINGS OF THE

AMERICAN MATHEMATICAL SOCIETY

Volume 135, Number 10, October 2007, Pages 3241-3247

S 0002-9939(07)08917-4

Article electronically published on June 20, 2007

\title{
ON LITTLEWOOD-PALEY FUNCTIONS
}

\author{
LESLIE C. CHENG \\ (Communicated by Michael T. Lacey)
}

\begin{abstract}
We prove that, for a compactly supported $L^{q}$ function $\Phi$ with vanishing integral on $\mathbf{R}^{n}$, the corresponding square function operator $S_{\Phi}$ is bounded on $L^{p}$ for $|1 / p-1 / 2|<\min \{(q-1) / 2,1 / 2\}$.
\end{abstract}

\section{INTRODUCTION}

Let $n \geq 1$ and $\mathbf{R}^{n}$ denote the $n$-dimensional Euclidean space. For a function $\Phi \in L^{1}\left(\mathbf{R}^{n}\right)$ which satisfies

$$
\int_{\mathbf{R}^{n}} \Phi(x) d x=0,
$$

we define the square function operator $S_{\Phi}$ by

$$
\left(S_{\Phi} f\right)(x)=\left(\int_{0}^{\infty}\left|\Phi_{t} * f(x)\right|^{2} \frac{d t}{t}\right)^{1 / 2}
$$

where $\Phi_{t}(x)=t^{-n} \Phi(x / t)$. The operator $S_{\Phi}$ is often called a square function or a Littlewood-Paley function. Such operators have long played important roles in harmonic analysis. The main problem under investigation concerns the boundedness of these operators on various $L^{p}$ spaces.

It has been well known that, if the function $\Phi$ is sufficiently nice (in terms of decaying and smoothness properties), the corresponding operator $S_{\Phi}$ is bounded on $L^{p}\left(\mathbf{R}^{n}\right)$ for $1<p<\infty$. The following result is due to Benedek, Calderón and Panzone ([2]):

Theorem A. Suppose that $\Phi$ satisfies (1.1) and for some positive $\alpha$,

$$
|\Phi(x)| \leq C(1+|x|)^{-n-\alpha}, \int_{\mathbf{R}^{n}}|\Phi(x-y)-\Phi(x)| d x \leq C|y|^{\alpha} .
$$

Then $S_{\Phi}$ is bounded on $L^{p}\left(\mathbf{R}^{n}\right)$ for $1<p<\infty$.

Examples of functions satisfying (1.3) include the Schwartz functions, as well as the following which arise from the Poisson kernel on $\mathbf{R}^{n}$ :

$$
\Phi_{0}(x)=\left.\frac{\partial}{\partial t}\left(\frac{t}{\left(|x|^{2}+t^{2}\right)^{(n+1) / 2}}\right)\right|_{t=1}
$$

Received by the editors June 27, 2006.

2000 Mathematics Subject Classification. Primary 42B25.

(C)2007 American Mathematical Society 
and

When $\Phi$ is given by

$$
\Phi_{j}(x)=\frac{\partial}{\partial x_{j}}\left(\frac{1}{\left(|x|^{2}+1\right)^{(n+1) / 2}}\right) .
$$

$$
\Phi(x)=|x|^{-n+1} \Omega(x) \chi_{[0,1]}(|x|),
$$

where $\Omega$ is homogeneous of degree 0 and has mean value zero on $\mathbf{S}^{n-1}$, then $S_{\Phi}$ becomes the Marcinkiewicz integral operator ([13). See also [1], 14, [16] and the extensive list of references given in the survey [6].

In 12 S. Sato proved that, among other things, the conclusion of Theorem A is still true if the smoothness condition (1.3) is eliminated (for $L^{2}$ this had been known earlier; see [5], [10]).

In this paper we shall study the $L^{p}$ boundedness of $S_{\Phi}$ without imposing conditions (1.2) or (1.3) on $\Phi$, or the assumption that $\Phi$ be given as in (1.4). The following is a known result:

Theorem B. Suppose that $\Phi$ satisfies (1.1) and is compactly supported.

(i) If $\Phi \in L^{q}\left(\mathbf{R}^{n}\right)$ for some $q \geq 2$, then $S_{\Phi}$ is a bounded operator on $L^{p}\left(\mathbf{R}^{n}, w\right)$ for $p>q^{\prime}$ and $w \in A_{p / q^{\prime}}$.

(ii) If $\Phi \in L^{2}\left(\mathbf{R}^{n}\right)$, then $S_{\Phi}$ is a bounded operator on $L^{p}\left(\mathbf{R}^{n}\right)$ for $1<p<\infty$.

In the above statement, $L^{p}\left(\mathbf{R}^{n}, w\right)$ represents the weighted $L^{p}$ space with weight $w$ (the definition of the weight class $A_{p}$ can be found in [11, 4] or [14). When $w \equiv 1$ we write $L^{p}\left(\mathbf{R}^{n}, w\right)$ as $L^{p}\left(\mathbf{R}^{n}\right)$.

Part (i) of Theorem B is due to S. Sato (Theorem 3 in [12]). Part (ii) follows from (i) by using duality and interpolation (with $w \equiv 1$ ).

Theorem B (ii) covers the cases $\Phi \in L^{q}\left(\mathbf{R}^{n}\right), q>2$ as well because of the compact support assumption. However, the approach used in [12] does not appear to work when $q<2$ (see also [9, page 241). The main purpose of the present paper is to establish the following theorem dealing with the case where $\Phi \in L^{q}\left(\mathbf{R}^{n}\right)$ for $q<2$.

Theorem C. Suppose that $\Phi$ is a compactly supported function satisfying (1.1). If $\Phi \in L^{q}\left(\mathbf{R}^{n}\right)$ for some $q \in(1,2]$, then $S_{\Phi}$ is a bounded operator on $L^{p}\left(\mathbf{R}^{n}\right)$ for $|1 / p-1 / 2|<(q-1) / 2$.

Remarks 1. (i) The range of $p$ given by $|1 / p-1 / 2|<(q-1) / 2$ is the same as $2 / q<p<2 /(2-q)$, which becomes $(1, \infty)$ when $q=2$.

(ii) A result relevant to the theorems mentioned above is Theorem 1 in [7. While in general an $L^{q}$ function $\Phi$ does not satisfy the pointwise decay condition imposed on its Fourier transform in Theorem 1 of [7, a modification of the proof given in [7] can yield the $L^{p}$ boundedness of $S_{\Phi}$ for $|1 / p-1 / 2|<(q-1) /(2 q)$ under the conditions in Theorem C. Since $q>1$, the range of $p$ given by $|1 / p-1 / 2|<(q-1) / 2$ in our theorem is considerably better.

(iii) While the inequality $|1 / p-1 / 2|<(q-1) / 2$ gives the full range $1<p<\infty$ when $q=2$, it would be an interesting problem to determine whether it also represents the best possible range for $p$ when $q<2$.

The proof of Theorem $\mathrm{C}$ will be given in Section 2. Section 3 contains a result on the boundedness of $S_{\Phi}$ when the compact support condition is replaced by some other conditions. 


\section{Proof of Theorem C}

Lemma 2.1. Let $\Psi$ be a compactly supported function in $L^{q}\left(\mathbf{R}^{n}\right)$ for some $q \in$ $(1,2]$. Then, for every $p$ satisfying $|1 / p-1 / 2|<(q-1) / 2$, there exists a $C_{p}>0$ such that

$$
\left\|\left(\int_{0}^{\infty}\left|\left(\Psi_{t} * F^{t}\right)(x)\right|^{2} \frac{d t}{t}\right)^{1 / 2}\right\|_{L^{p}\left(\mathbf{R}^{n}\right)} \leq C_{p}\left\|\left(\int_{0}^{\infty}\left|F^{t}(x)\right|^{2} \frac{d t}{t}\right)^{1 / 2}\right\|_{L^{p}\left(\mathbf{R}^{n}\right)}
$$

holds for every measurable function $F^{t}(x)=F(t, x)$ on $(0, \infty) \times \mathbf{R}^{n}$.

Proof. By duality and interpolation we may assume that $p>2$. We shall also assume that $\operatorname{supp}(\Psi)$ is contained in $B(0,1)$, where $B\left(x_{0}, t\right)=\left\{x \in \mathbf{R}^{n}:\left|x-x_{0}\right| \leq\right.$ $t\}$. Let $T$ be the operator acting on functions defined on $(0, \infty) \times \mathbf{R}^{n}$ given by

$$
T(F)(t, x)=\left(\Psi_{t} * F^{t}\right)(x),
$$

where $F^{t}(y)=F(t, y)$ for $(t, y) \in(0, \infty) \times \mathbf{R}^{n}$. For $1 \leq p, q<\infty$ we shall use the following notation:

$$
\|F\|_{L^{p}\left(L^{q}\left(t^{-1} d t\right), d x\right)}=\left(\int_{\mathbf{R}^{n}}\left(\int_{0}^{\infty}|F(t, x)|^{q} \frac{d t}{t}\right)^{p / q} d x\right)^{1 / p}
$$

Thus we have

$$
\begin{gathered}
\|T(F)\|_{L^{1}\left(L^{1}\left(t^{-1} d t\right), d x\right)}=\int_{\mathbf{R}^{n}}\left(\int_{0}^{\infty}\left|\left(\Psi_{t} * F^{t}\right)(x)\right| t^{-1} d t\right) d x \\
=\int_{0}^{\infty}\left\|\Psi_{t} * F^{t}\right\|_{L^{1}\left(\mathbf{R}^{n}\right)} t^{-1} d t \\
\leq\|\Psi\|_{L^{1}\left(\mathbf{R}^{n}\right)} \int_{0}^{\infty}\left\|F^{t}\right\|_{L^{1}\left(\mathbf{R}^{n}\right)} t^{-1} d t \\
=\|\Psi\|_{L^{1}\left(\mathbf{R}^{n}\right)}\|F\|_{L^{1}\left(L^{1}\left(t^{-1} d t\right), d x\right)}
\end{gathered}
$$

It follows from $1 / 2-1 / p<(q-1) / 2$ that

$$
p^{\prime} q / 2>1 \text {. }
$$

Let $r=\left(p^{\prime} q / 2\right)^{\prime}$. By $p>2$ we have $r>q^{\prime}$. Thus, for any $F$ that satisfies

$$
\|T(F)\|_{L^{r}\left(L^{\left.q^{\prime}\left(t^{-1} d t\right), d x\right)}\right.}<\infty,
$$

there exists a function $h \in L^{\left(r / q^{\prime}\right)^{\prime}}\left(\mathbf{R}^{n}\right)$ such that

$$
\|h\|_{L^{\left(r / q^{\prime}\right)^{\prime}\left(\mathbf{R}^{n}\right)}}=1
$$

and

$$
\|T(F)\|_{L^{r}\left(L^{q^{\prime}}\left(t^{-1} d t\right), d x\right)}^{q^{\prime}}=\int_{\mathbf{R}^{n}}\left(\int_{0}^{\infty}\left|\left(\Psi_{t} * F^{t}\right)(x)\right|^{q^{\prime}} t^{-1} d t\right) h(x) d x .
$$

By Hölder's inequality,

$$
\begin{aligned}
& \left|\Psi_{t} * F^{t}(x)\right|^{q^{\prime}}=t^{-n q^{\prime}}\left|\int_{\mathbf{R}^{n}} \Psi\left(\frac{x-y}{t}\right) F(t, y) d y\right|^{q^{\prime}} \\
\leq & \|\Psi\|_{L^{q}\left(\mathbf{R}^{n}\right)}^{q^{\prime}}|B(0, t)|^{-1} \int_{\mathbf{R}^{n}}|F(t, y)|^{q^{\prime}} \chi_{B(0, t)}(x-y) d y .
\end{aligned}
$$


Let $M$ denote the Hardy-Littlewood maximal operator on $\mathbf{R}^{n}$. Then by (2.5) and $(2.6)$,

$$
\begin{aligned}
& \|T(F)\|_{L^{r}\left(L^{\left.q^{\prime}\left(t^{-1} d t\right), d x\right)}\right.}^{q^{\prime}} \leq\|\Psi\|_{L^{q}\left(\mathbf{R}^{n}\right)}^{q^{\prime}} \int_{\mathbf{R}^{n}}\left(\int_{0}^{\infty}|F(t, y)|^{q^{\prime}} t^{-1} d t\right)(M h)(y) d y \\
& \leq\|\Psi\|_{L^{q}\left(\mathbf{R}^{n}\right)}^{q^{\prime}}\left\|\left(\int_{0}^{\infty}|F(t, y)|^{q^{\prime}} t^{-1} d t\right)\right\|_{L^{\left(r / q^{\prime}\right)}\left(\mathbf{R}^{n}\right)}^{q^{\prime}}\|M h\|_{L^{\left(r / q^{\prime}\right)^{\prime}\left(\mathbf{R}^{n}\right)}} \\
& \leq C_{r, q}\|\Psi\|_{L^{q}\left(\mathbf{R}^{n}\right)}^{q^{\prime}}\|F\|_{L^{r}\left(L^{\left.q^{\prime}\left(t^{-1} d t\right), d x\right)}\right.}^{q^{\prime}} .
\end{aligned}
$$

Since

$$
\begin{aligned}
& \frac{1}{2}=\frac{\theta}{q^{\prime}}+\frac{(1-\theta)}{1}, \\
& \frac{1}{p}=\frac{\theta}{r}+\frac{(1-\theta)}{1}
\end{aligned}
$$

hold with $\theta=q / 2$, by interpolating between (2.4) and (2.7) (see, for example, [3]) we obtain

$$
\|T(F)\|_{L^{p}\left(L^{2}\left(t^{-1} d t\right), d x\right)} \leq C_{p}\|F\|_{L^{p}\left(L^{2}\left(t^{-1} d t\right), d x\right)}
$$

which proves (2.1) for $|1 / p-1 / 2|<(q-1) / 2$.

For $s>0$ we let $s^{ \pm \alpha}=\min \left\{s^{\alpha}, s^{-\alpha}\right\}$.

Lemma 2.2. Let $\Psi \in \mathcal{S}\left(\mathbf{R}^{n}\right)$ and $\Phi \in L^{q}\left(\mathbf{R}^{n}\right)$ for some $q \in(1,2]$. Suppose that $\Phi$ is compactly supported and $\operatorname{supp}(\widehat{\Psi}) \subset\{1 / 2<|\xi|<2\}$. Then for every $p$ satisfying $|1 / p-1 / 2|<(q-1) / 2$, there exist $C_{p}, \alpha_{p}>0$ such that

$$
\left\|\left(\int_{0}^{\infty}\left|\Phi_{t} * \Psi_{s t} * f\right|^{2} \frac{d t}{t}\right)^{1 / 2}\right\|_{L^{p}\left(\mathbf{R}^{n}\right)} \leq C_{p}\left(s^{ \pm \alpha_{p}}\right)\|f\|_{L^{p}\left(\mathbf{R}^{n}\right)}
$$

for all $f \in L^{p}\left(\mathbf{R}^{n}\right)$ and $s>0$.

Proof. Let

$$
T_{s} f(x)=\left(\int_{0}^{\infty}\left|\Phi_{t} * \Psi_{s t} * f(x)\right|^{2} \frac{d t}{t}\right)^{1 / 2} .
$$

Then, by Lemma 2.1 and Theorem A, for each $p$ satisfying $|1 / p-1 / 2|<(q-1) / 2$,

$$
\begin{gathered}
\left\|T_{s} f\right\|_{L^{p}\left(\mathbf{R}^{n}\right)} \leq C_{p}\left\|S_{\Psi} f\right\|_{L^{p}\left(\mathbf{R}^{n}\right)} \\
\leq C_{p}\|f\|_{L^{p}\left(\mathbf{R}^{n}\right)} .
\end{gathered}
$$

On the other hand, by Plancherel's Theorem,

$$
\begin{gathered}
\left\|T_{s} f\right\|_{L^{2}\left(\mathbf{R}^{n}\right)}^{2}=\int_{0}^{\infty} \int_{\mathbf{R}^{n}}\left|\Phi_{t} * \Psi_{s t} * f(x)\right|^{2} d x t^{-1} d t \\
=\int_{\mathbf{R}^{n}}|\hat{f}(\xi)|^{2}\left(\int_{0}^{\infty}|\widehat{\Phi}(t \xi)|^{2}|\widehat{\Psi}(s t \xi)|^{2} \frac{d t}{t}\right) d \xi .
\end{gathered}
$$

It follows from Lemmas 2, 3 of [12] and (1.1) that

$$
\int_{1 / 2}^{2}|\widehat{\Phi}(t \xi)|^{2} d t \leq C|\xi|^{ \pm 1 /\left(2 q^{\prime}\right)}
$$


Let $\xi^{\prime}=|\xi|^{-1} \xi$ for $\xi \neq 0$. By (2.10) and (2.11) we have

$$
\begin{aligned}
\left\|T_{s} f\right\|_{L^{2}\left(\mathbf{R}^{n}\right)}^{2} & \leq \int_{\mathbf{R}^{n}}|\hat{f}(\xi)|^{2}\left(\int_{1 / 2}^{2}\left|\widehat{\Phi}\left(t s^{-1} \xi^{\prime}\right)\right|^{2} \frac{d t}{t}\right) d \xi \\
& \leq C\left(s^{ \pm 1 /\left(2 q^{\prime}\right)}\right)\|f\|_{L^{2}\left(\mathbf{R}^{n}\right)}^{2} .
\end{aligned}
$$

By (2.9), (2.12) and interpolation we conclude that (2.8) holds for $|1 / p-1 / 2|<$ $(q-1) / 2$.

Proof of Theorem C. It suffices to establish

$$
\left\|S_{\Phi} f\right\|_{L^{p}\left(\mathbf{R}^{n}\right)} \leq C_{p}\|f\|_{L^{p}\left(\mathbf{R}^{n}\right)}
$$

for $f \in \mathcal{S}\left(\mathbf{R}^{n}\right)$ and $|1 / p-1 / 2|<(q-1) / 2$.

Let $\eta \in C^{\infty}(\mathbf{R})$ such that $\operatorname{supp}(\eta) \subset(1 / 4,4)$ and

$$
\int_{0}^{\infty} \eta(s) \frac{d s}{s}=2 .
$$

Define the Schwartz function $\Psi$ on $\mathbf{R}^{n}$ by

$$
\widehat{\Psi}(\xi)=\eta\left(|\xi|^{2}\right)
$$

for $\xi \in \mathbf{R}^{n}$. Then by (2.13) we have

$$
\int_{0}^{\infty} \widehat{\Psi}(s \xi) \frac{d s}{s}=1
$$

and

$$
\Phi_{t} * f=\int_{0}^{\infty}\left(\Phi_{t} * \Psi_{s t} * f\right) \frac{d s}{s}
$$

for all $f \in \mathcal{S}\left(\mathbf{R}^{n}\right)$ and $t>0$. By Minkowski's inequality we have

$$
\left|S_{\Phi} f(x)\right| \leq \int_{0}^{\infty}\left(\int_{0}^{\infty}\left|\Phi_{t} * \Psi_{s t} * f(x)\right|^{2} \frac{d t}{t}\right)^{1 / 2} \frac{d s}{s} .
$$

By Lemma 2.2, for every $p$ satisfying $|1 / p-1 / 2|<(q-1) / 2$,

$$
\begin{gathered}
\left\|S_{\Phi} f\right\|_{L^{p}\left(\mathbf{R}^{n}\right)} \leq C_{p}\|f\|_{L^{p}\left(\mathbf{R}^{n}\right)} \int_{0}^{\infty} s^{ \pm \alpha_{p}} \frac{d s}{s} \\
=2 \alpha_{p}^{-1} C_{p}\|f\|_{L^{p}\left(\mathbf{R}^{n}\right)} .
\end{gathered}
$$

The proof of Theorem $\mathrm{C}$ is now complete.

\section{Further RESUlts}

For $\Phi$ 's which are not necessarily compactly supported, one can easily deduce the following result from Theorem 2 of [12] by using duality and interpolation:

Theorem 3.1. Let $\Phi \in L^{1}\left(\mathbf{R}^{n}\right)$ and satisfy (1.1). Suppose that

$$
\int_{|x|<1}|\Phi(x)|^{1+\varepsilon} d x+\int_{|x|>1}|\Phi(x)||x|^{\varepsilon} d x<\infty \text { for some } \varepsilon>0
$$

(ii) $\quad|\Phi(x)| \leq h(|x|) \Omega\left(x^{\prime}\right)$, where

(ii.a) $h$ is non-negative, non-increasing and satisfies $\int_{0}^{\infty} h(r) r^{n-1} d r<\infty$;

(ii.b) $\Omega \in L^{q}\left(\mathbf{S}^{n-1}\right)$ for some $q \geq 2$.

Then $S_{\Phi}$ is bounded on $L^{p}\left(\mathbf{R}^{n}\right)$ for $1<p<\infty$. 
Using the above theorem one can see that the condition (1.4) in Theorem A is redundant, as observed in [12].

Below we shall show that the requirement " $q \geq 2$ " in (ii.b) of the above theorem can be lowered to $q>1$ without affecting the validity of the claim.

Theorem 3.2. If the condition (ii.b) in Theorem 3.1 is replaced by the weaker condition (ii.b)': $\Omega \in L^{q}\left(\mathbf{S}^{n-1}\right)$ for some $q>1$, while all other conditions in Theorem 3.1 remain unchanged, then $S_{\Phi}$ is bounded on $L^{p}\left(\mathbf{R}^{n}\right)$ for $1<p<\infty$.

Proof. By Lemmas 1-3 in [12], we see that (2.11) still holds (note that $q>1$ is needed when applying Lemma 2 of [12]). Thus, it suffices to show that (2.1) holds for all $p \in(1, \infty)$.

For each $y^{\prime} \in \mathbf{S}^{n-1}$ and $x \in \mathbf{R}^{n}$, let

$$
M_{y^{\prime}} f(x)=\sup _{s>0}\left(\frac{1}{s} \int_{0}^{s}\left|f\left(x-s y^{\prime}\right)\right| d s\right) .
$$

For $t>0$ and $x \in \mathbf{R}^{n}$,

$$
\begin{gathered}
\left|\Phi_{t} * f(x)\right| \leq \int_{\mathbf{S}^{n-1}}\left|\Omega\left(y^{\prime}\right)\right|\left(\int_{0}^{\infty}\left|f\left(x-t r y^{\prime}\right)\right| h(r) r^{n-1} d r\right) d \sigma\left(y^{\prime}\right) \\
\leq \int_{\mathbf{S}^{n-1}}\left|\Omega\left(y^{\prime}\right)\right|\left(\sum_{j=-\infty}^{\infty} 2^{n(j+1)} h\left(2^{j}\right) M_{y^{\prime}} f(x)\right) d \sigma\left(y^{\prime}\right) \\
\leq 2^{2 n}\left(\int_{0}^{\infty} h(r) r^{n-1} d r\right) \int_{\mathbf{S}^{n-1}}\left|\Omega\left(y^{\prime}\right)\right| M_{y^{\prime}} f(x) d \sigma\left(y^{\prime}\right) .
\end{gathered}
$$

By (3.1) and the uniform boundedness of the operators $M_{y^{\prime}}$ on $L^{p}\left(\mathbf{R}^{n}\right)$ for $1<p<$ $\infty$, we have

$$
\left\|\sup _{t>0}\left|\Phi_{t} * f\right|\right\|_{L^{p}\left(\mathbf{R}^{n}\right)} \leq C_{p}\|\Omega\|_{1}\|f\|_{L^{p}\left(\mathbf{R}^{n}\right)}
$$

for $1<p<\infty$. It follows from (3.2) and the proof of the lemma on p. 544 of 8 (after some trivial modifications) that $(2.1)$ holds for $1<p<\infty$.

\section{REFERENCES}

[1] Al-Salman, A., Al-Qassem, H., Cheng, L. C., Pan, Y., $L^{p}$ Bounds for the Function of Marcinkiewicz, Math. Res. Lett. 9 (2002), 697-700. MR.1906071 (2004d:42020)

[2] Benedek, A., Calderón, A. and Panzone, R., Convolution operators on Banach space valued functions, Proc. Natl. Acad. Sci. USA 48 (1962), 356-365. MR0133653 (24:A3479)

[3] Bergh, J. and Löfström, J., Interpolation Spaces, Springer-Verlag, 1976.

[4] Coifman, R. and Fefferman, C., Weighted norm inequalities for maximal functions and singular integrals, Studia Math. 51 (1974), 241-250. MR0358205 (50:10670)

[5] Coifman, R. and Meyer, Y., Au delà des opérateurs pseudo-différentiels, Astérisque $\mathbf{5 7}$ (1978). MR518170 (81b:47061)

[6] Ding, Y., On Marcinkiewicz integral, Proc. of the conference "Singular Integrals and Related Topics, III", Osaka, Japan (2001), 28-38.

[7] Ding, Y., Fan, D.. and Pan, Y., On Littlewood-Paley functions and singular integrals, Hokkaido Math. J. 29 (2000), 537-552. MR.1795491 (2001m:42038)

[8] Duoandikoetxea, J. and Rubio de Francia, J. L., Maximal and singular integral operators via Fourier transform estimates, Invent. Math. 84 (1986), 541-561. MR837527 (87f:42046)

[9] Duoandikoetxea, J. and Seijo, E., Weighted inequalities for rough square functions through extrapolation, Studia Math. 149 (2002), 239-252. MR1890732 (2003a:42022)

[10] Journé, J.-L., Calderón-Zygmund operators, pseudo-differential operators and the Cauchy integral of Calderón, Lecture Notes in Math. 994 (1983), Springer-Verlag. MR706075 (85i:42021) 
[11] Muckenhoupt, B., Weighted norm inequalities for the Hardy maximal functions, Trans. Amer. Math. Soc. 165 (1972), 207-226. MR0293384 (45:2461)

[12] Sato, S., Remarks on square functions in the Littlewood-Paley theory, Bull. Austral. Math. Soc. 58 (1998), 199-221. MR1642027 (99g:42024)

[13] Stein, E.M., On the functions of Littlewood-Paley, Lusin and Marcinkiewicz, Trans. Amer. Math. Soc. 88 (1958), 430-466. MR0112932 (22:3778)

[14] Stein, E.M., Harmonic Analysis: Real-variable methods, orthogonality, and oscillatory integrals, Princeton University Press, 1993. MR.1232192 (95c:42002)

[15] Stein, E.M., Singular integrals and differentiability properties of functions, Princeton University Press, 1970. MR0290095 (44:7280)

[16] Walsh, T., On the function of Marcinkiewicz, Studia Math. 44 (1972), 203-217. MR0315368 $(47: 3917)$

Department of Mathematics, Bryn Mawr College, Bryn Mawr, Pennsylvania 19010

E-mail address: lcheng@brynmawr.edu 